\title{
INTERACTIONS OF HEMIN, ANTIMALARIAL DRUGS AND HEMIN-ANTI- MALARIAL COMPLEXES WITH PHOSPHOLIPID MONOLAYERS
}

\author{
HAGAI GINSBURG ${ }^{a}$ and RUDY A. DEMEL ${ }^{b}$

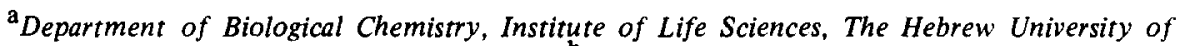 \\ Jerusalem, 91904 Jerusalem (Israel) and biochemistry Laboratory, State University of \\ Utrecht, Padualaan 8, $3584 \mathrm{CH}$ Utrecht (The Netherlands)
}

Received December 12 th, 1983 accepted July 3rd, 1984

Hemin, antimalarial drugs and complexes formed between them, have demonstrable effects on biological membranes. Using the phospholipid monolayer model, we show that hemin intercalates into the membrane and increases its surface pressure, depending on the lipid composition and the initial surface pressure: negative surface charges and particularly looser compaction of the phospholipids reduce the effect of hemin. With increasing surface pressure hemin tends to intercalate as a monomer, and the half-saturation concentration of its effect increases exponentially. The antimalarial monovalent drugs quinine and mefloquine, but not chloroquine, also penetrate into the membrane and expand it. All three drugs markedly increase the effect of hemin, but chloroquine reduces the effect in monolayers composed of unsaturated phospholipids. The drugs' effect is mostly due to an increase in the maximal surface pressure and suggests a complexation of hemin and drug within the membrane phase. Preformed hemindrug complexes decrease the half-saturation concentration of the effect and suggest that the complexes adsorb to the membrane, releasing the hemin through an apolar continuum into the phospholipid phase. The implications of the results to the membrane toxicity mechanism proposed for the molecular mode of action of antimalarial drugs are discussed.

Keywords: phospholipids; monolayers; hemin; antimalarial drugs.

\section{Introduction}

Hemin can cause lysis of mouse [1,2] and human erythrocytes [3] and of malaria parasites $[4,5]$. Other types of cells were demonstrably resistant to this lytic effect (see Ref. 6, for example). Although the molecular details of hemininduced lysis are not fully understood, the lipid constituents of cellular membranes have been invoked as the hemin receptors [3] and hemin binding to and crossing through phospholipid bilayers is well established $[7,8]$. We have recently shown [9] that phospholipid monolayers serve as an instructive model for the study of hemin interaction with lipid membranes and, based on our findings, we suggested that this interaction depends both on the nature of the phospholipid headgroup and the degree of saturation of its acyl chains.

A totally different class of compound, e.g., cationic amphiphile, including 
efficient antimalarial drugs, also bind to phospholipids (see Ref. 10 for a review; also Refs. 11-13). This binding depends on the charge of the headgroup and the hydrophobicity of the amphiphile [14] and it has been invoked in lysosomotropic effects and in lipidosis caused by these drugs $[10,15]$. The combined toxic effect of hemin and the antimalarial drug chloroquine on cell membranes has been suggested to underly the pharmacological mode of action of this drug [16]. Hemin forms complexes with antimalarial drugs both in aqueous $[1,17]$ and apolar [18] phases and in the presence of these complexes erythrocytes and malaria parasites lyse $[1,4,5]$. These observations, in conjunction with the present resurgence of malaria which is mostly due to chloroquine resistance and the lack of cross resistance to other 4 -aminoquinolines $[19,20]$, underscores the necessity for further probing of the molecular details of the interaction of hemin, drugs and hemin-drug complexes with phospholipids. In the present work we used the phospholipid monolayer system [21] and tested their interactions using different compositions of phospholipids and the three antimalarial 4-aminoquinoline drugs: chloroquine, quinine and mefloquine.

\section{Materials and Methods}

\section{Chemicals}

Phosphatidylserine (PS) from bovine brain, cholesterol (CHOL), chloroquinediphosphate (CQ) and ferriprotophyrin IX (equine hemin, FP), were purchased from Sigma; phosphatidylinositol (PI) from yeast, from Koch-Light; quinine sulfate (Q) was from Aldrich.

Mefloquine-hydrochloride (WR-142, 490, MQ-1) and its threo-anomer (WR-177, 602, MQ-2) were donated by The Walter Reed Army Research Institute. Where MQ alone appears in the text, it refers to MQ-1. All other chemicals used were of the highest grade available.

Other phospholipids used in this study were synthesized [22,23] and purified by high pressure liquid chromatography [35]. These were: 1,2-dilinoleoyl-snphosphatidylcholine (DLPC), 1,2-dioleoyl-sn-phosphatidylcholine (DOPC) and 1,2-dipalmitoyl-sn-phosphatidylcholine (DPPC).

\section{Solutions}

$\mathrm{CQ}$ and $\mathrm{Q}$ were prepared to $1 \mathrm{mM}$ in water. MQ-1 and MQ-2 were dissolved in ethanol/water $(1: 9)$ at $1 \mathrm{mM}$. All phospholipids were dissolved at $\sim 1 \mathrm{mM}$ in chloroform/methanol $(9: 1)$. The aqueous subphase consisted invariably of Trisbuffered saline: $10 \mathrm{mM}$ Tris $-\mathrm{HCl}, 150 \mathrm{mM} \mathrm{NaCl}(\mathrm{pH} \mathrm{7.4)}$ at room temperature.

Fresh hemin stock solutions $(1 \mathrm{mM})$ were prepared daily by dissolving a weighed amount in $\mathrm{NaOH}, 0.02 \mathrm{~N}$, at room temperature. Solubilization was complete in $1.5-2 \mathrm{~h}$ and the precise concentration was checked spectrophotometrically $\left(\epsilon^{385}=\right.$ $6.1 \times 10^{4} \mathrm{M} \mathrm{cm}^{-1}$ ). The solution was thereafter kept in the dark on ice until use. 


\section{Measurements of surface pressure}

Phospholipid monolayers were formed at the air-water interface in a Teflon trough $33.2 \mathrm{~cm}^{2}$ surface area, $19 \mathrm{ml}$ volume of aqueous phase). Formation and stabilization of the monolayer were followed by measuring the surface pressure $(\pi)$ by means of a Wilhelmy plate and an electronic microbalance [21]. The initial pressure $\pi_{i}$ was recorded; then various compounds, e.g., FP, CQ, Q, etc., were added as detailed in Results and the change in surface pressure $\Delta \pi$ was measured after steady state was achieved. The measuring system was calibrated using the surface pressure of pure water. In most cases this steady state reflected true thermodynamic equilibrium, inasmuch as no further changes in surface pressure could be observed with time. In some experimental conditions, e.g., addition of preformed drug-hemin complexes, true equilibrium could not be achieved, even after $6-7 \mathrm{~h}$. Longer incubation times, however, were compromised by non-specific processes such as leakage of materials from the monolayer and possible evaporation, and therefore these results should be considered only as quasi-steady state rather than true equilibrium.

\section{Analysis of data}

Since $\Delta \pi$ depends on $\pi_{i}[34]$, monolayers were formed at different $\pi_{i}$ 's, titrated with additive, and $\Delta \pi$ was recorded. Data obtained at the same titrant concentration displayed a linear dependence of $\Delta \pi$ on $\pi_{i}$ and were, therefore, analyzed by linear regression, thus obtaining an intercept (l) and a slope (S) for the FP concentration (C). Subsequent analysis of the dependence of $I$ and $S$ on $C$ yielded a relationship of the general form:

$$
\mathrm{I}=\mathrm{I}_{\max } \cdot \mathrm{C}^{x} /\left(\mathrm{K}_{\mathrm{I}}+C^{x}\right) \text { and } \mathrm{S}=\mathrm{S}_{\max } \cdot \mathrm{C}^{y} /\left(\mathrm{K}_{\mathrm{S}}+\mathrm{C}^{y}\right)
$$

The different parameters were retrieved by non-linear, least squares analysis of the data.

Next, the dependence of $\Delta \pi$ on $C$ for any $\pi_{i}$ chosen was generated using the following equation:

$$
\Delta \pi=\mathrm{I}_{\max } \cdot \mathrm{C}^{x} /\left(\mathrm{K}_{\mathrm{I}}+\mathrm{C}^{x}\right)-\pi_{i} \cdot \mathrm{S}_{\max } \cdot \mathrm{C}^{x} /\left(\mathrm{K}_{\mathrm{S}}+\mathrm{C}^{y}\right)
$$

Finally, the generated data were analyzed by non-linear, least squares analysis to fit the following equation:

$$
\Delta \pi=\Delta \pi_{\max } \cdot \mathrm{C}^{z} /\left(\mathrm{K}_{z}+\mathrm{C}^{z}\right)
$$

\section{Results}

\section{Effect of FP on surface pressure}

Figure 1 shows a typical experiment assaying the effect of FP on $\Delta \pi$. Mono- 


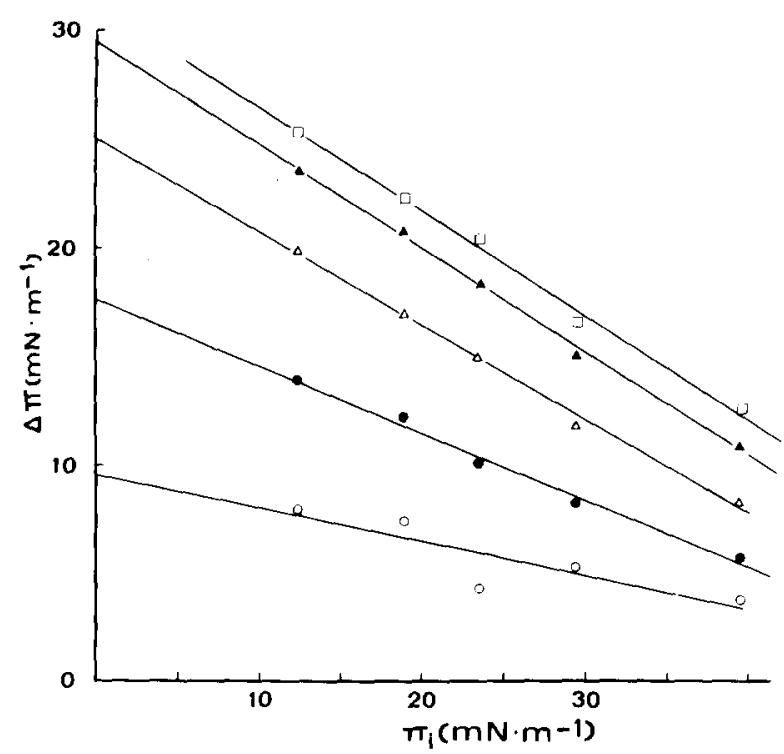

Fig. 1. Effect of hemin on the surface pressure of DOPC monolayers. Monolayers of DOPC were formed at different initial surface pressures $\left(\pi_{i}\right)$, and titration with a stock solution of hemin (FP) was performed, waiting after each addition for the stabilization of $\pi$. Equal concentration points were connected by lines drawn according to the parameters derived from linear regression analysis. FP concentrations $(\mu \mathrm{M}): \circ, 0.263 ; \bullet, 0.526 ;\lrcorner, 1.053 ; \bullet, 2.105$; $\square, 3.68$.

layers of DOPC were formed at various $\pi_{i}$ 's and increasing amounts of FP were added from stock solution to the aqueous subphase, allowing for stable reading after each addition and recording $\Delta \pi$. Experimental results were highly reproducible, provided the Wilhelmy plate and the Teflon trough were meticulously cleaned between two consecutive experiments. The observed increase in $\pi$ is certainly due to the intercalation of FP into the monolayer. The fact that all the experimental values of $\Delta \pi$ obtained with the same FP concentration, but with different monolayers having different $\pi_{i}$ 's, regress into a single straight line with a high correlation coefficient, is an intrinsic control for the validity and reproducibility of the measurements.

Similar experiments were performed on monolayers formed from the following molar mixtures of lipids: DOPC/PS (1:1), DOPC/PI $(1: 1)$ and DOPC/CHOL $(2: 1)$. The slopes and intercepts for each concentration of FP were obtained by least squares linear regression, and the dependence of the slope and intercept on FP concentration was analyzed by non-linear, least squares fitting according to Eqn. (1), using the Levenberg-Marquand method. Results shown in Table I demonstrate, in most cases, the high accuracy of the measurements as evidenced by the relatively small statistical scatter of the data. 


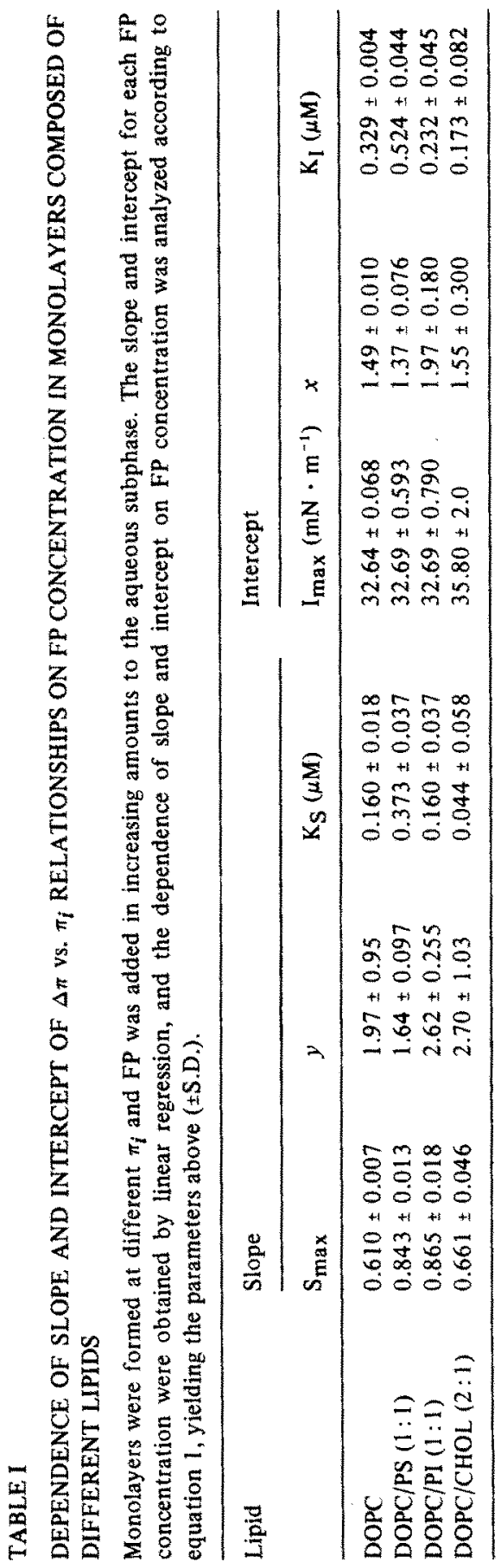


Comparison of $\Delta \pi$ generated in different types of monolayers by a given concentration of FP at equal $\pi_{i}$ (Table II), indicate that the effect of FP on $\Delta \pi$ depends on the type of phospholipid used. With phosphatidylcholines the effect increased with the degree of saturation of the fatty acid chains, i.e. with denser packing of the phospholipids in the monolayer and/or with its greater hydrophobicity. The greatest effect was observed in monolayers containing cholesterol which demonstrably causes larger packing of monolayers [25].

In the presence of the acidic phospholipids PS and PI, the effect of FP on $\pi$ is much less pronounced. These results suggest that the negative surface charge of the monolayer reduces the partitioning of the negatively charged FP into the monolayers, and imply a considerable electrostatic effect in this partitioning.

\section{Effects of antimalarial drugs on surface pressure}

Three antimalarial drugs were tested in the present investigation, namely: chloroquine (CQ), quinine (Q) and mefloquine (MQ). All are 4-aminoquinolines with different side chains and weak base properties; at neutral $\mathrm{pH}$ they are positively charged. Most of the $\mathrm{CQ}$ is diprotonated at this $\mathrm{pH}\left(\mathrm{pK}_{\mathrm{b} 1}=10.2: \mathrm{pK}_{\mathrm{b} 2}=8.3\right)$ and, although it has been shown to bind to neutral and acid phospholipids $[11,26]$, no effect on the surface pressure of DOPC and DOPC-PS monolayers was observed. Quinine is monoprotonated $\left(\mathrm{pK}_{\mathrm{b}}=8.35\right)$ and has no effect on DOPC monolayers. With DOPC-PS monolayers it increases the surface pressure at $4.1 \mathrm{mN} \cdot \mathrm{m}^{-1}$ per $\mu \mathrm{M}$ with no apparent saturation (up to $10 \mu \mathrm{M}$ ). Mefloquine is a quinoline methanol with a piperidine side chain, which binds to phosphatidylcholine and, with higher affinity and to a greater extent, to PS [26]. Commensurately, it was

\section{TABLE II}

EFFECT OF FP $(0.5 \mu \mathrm{M})$ ON $\Delta \pi$ OF MONOLAYERS COMPOSED OF DIFFERENT LIPIDS AT IDENTICAL $\pi_{i}\left(24 \mathrm{mN} \cdot \mathrm{m}^{-1}\right)$

N.D., not determined. Data given in the left column were calculated using parameters given in Table I and Eqn. (2). Data shown in the right column were obtained by direct measurement of $\Delta \pi$ produced in monolayers initially formed at $\pi_{i}=24 \mathrm{mN} \cdot \mathrm{m}^{-1}$ upon addition of $0.526 \mu \mathrm{M}$ FP. These are the means of triplicates, S.D. being $\leq 10 \%$ of the means.

\begin{tabular}{lcc}
\hline Lipid & $\begin{array}{l}\text { Calculated } \Delta \pi \\
\left(\mathrm{mN} \cdot \mathrm{m}^{-1}\right)\end{array}$ & $\begin{array}{l}\text { Measured } \Delta \pi \\
\left(\mathrm{mN} \cdot \mathrm{m}^{-1}\right)\end{array}$ \\
\hline DPPC & N.D. & 15.0 \\
DOPC & 8.19 & 6.9 \\
DLPC & N.D. & 7.3 \\
DOPC/PS $(1: 1)$ & 4.63 & 4.7 \\
DOPC/PI $(1: 1)$ & 6.54 & 4.6 \\
DOPC/CHOL $(2: 1)$ & 12.05 & $7.9^{\mathrm{a}}$ \\
\hline
\end{tabular}

${ }^{\mathrm{a}} \mathrm{FP}=0.26 \mu \mathrm{M}$. 
found to intercalate into DOPC monolayers with $\mathrm{K}_{1 / 2}=2.0 \pm 0.45 \mu \mathrm{M}$ and $\Delta \pi_{\max }$ $=4.8 \pm 0.9 \mathrm{mN} \cdot \mathrm{m}^{-1}$ and to the negatively charged DOPC-PS monolayers with $\mathrm{K}_{1 / 2}=1.1 \pm 0.25 \mu \mathrm{M}$ and $\Delta \pi_{\max }=6.47 \pm 0.56 \mathrm{mN} \cdot \mathrm{m}^{-1}$, implicating the importance of electrostatic interactions.

\section{The combined effect of FP and drugs on $\Delta \pi$}

In preliminary experiments we found that the order of addition of FP and drugs to the aqueous subphase affects the quasi-steady state of surface pressure. This was indeed expected from the fact that all the drugs produce multimeric complexes with FP in aqueous solutions $[1,27-29]$ and $Q$ and MQ interact with FP in apolar environment [18]. Thus, two different experimental procedures were utilized: either the drug was added first and then FP or a mixture of FP and drug was preformed and then added. All results were normalized to an arbitrary $\pi_{i}$ of $24 \mathrm{mN}$. $\mathrm{m}^{-1}$, using the following correction: Let

$$
\frac{I_{\max } \cdot C^{x}}{K_{I}+C^{x}}=A \quad \text { and } \quad \frac{S_{\max } \cdot C^{y}}{K_{S}+C^{y}}=B
$$

then

$$
\Delta \pi^{\prime}=\mathrm{A}-\pi_{i}^{\prime} \cdot \mathrm{B} \quad \text { and } \quad \Delta \pi_{24}=\mathrm{A}-24 \cdot \mathrm{B}
$$

where $\Delta \pi^{\prime}$ is the measured increase in surface pressure above the initial value of $\pi_{i}^{\prime}$ and $\Delta \pi_{24}$ is the corrected value for $\pi_{i}=24 \mathrm{mN} \cdot \mathrm{m}^{-1}$. By rearrangement and substitution we get

$$
\Delta \pi_{24}=\Delta \pi^{\prime}+\mathrm{B}\left(\pi_{i}^{\prime}-24\right)
$$

Values of $\mathrm{S}_{\max }, y$ and $\mathrm{K}_{\mathrm{S}}$ were taken from Table $\mathrm{I}$ for the calculation of $\mathrm{B}$. The dependence of $\Delta \pi_{24}$ on [FP] was analyzed by non-linear regression to fit Eqn. (3).

Results shown in Table III indicate that with DOPC monolayers the effect of the drug is exclusively on $\Delta \pi_{\max }$, suggesting that the drug does not affect the affinity of FP to the monolayers and probably forms complexes with FP in the monolayer, allowing more FP to distribute into the membrane and therefore increasing $\Delta \pi_{\max }$. With $\mathrm{CQ}$ the equilibration time was very long $\left(t_{1 / 2}>50 \mathrm{~min}\right)$ as compared to $\mathrm{Q}$ and $\mathrm{MQ}\left(t_{1 / 2} \sim 2-3 \mathrm{~min}\right)$, and on the time scale of $4-8 \mathrm{~min}$, the effect of CQ on $\Delta \pi$ was almost nil. After all free CQ was complexed with FP, further addition of FP caused an increase in $\Delta \pi$, as though the drug were absent (Fig. 2). By extrapolation of the linear part of the titration curve to $\Delta \pi=0$, it is possible to estimate a stoichiometry of the drug.FP complex of $1: 2[27,28]$. Similar results were obtained with $C Q$ in DOPC-PS monolayers (not shown), but a different picture emerges from the data of $\mathrm{Q}$ and $\mathrm{MQ}$ obtained with these monolayers (Table III). Here the major effect of the drug is to reduce $\mathrm{K}_{z}$ as it should be 


\section{TABLE III}

\section{EFFECT OF ANTIMALARIAL DRUGS AND FP ON $\Delta \pi$}

Monolayers were formed with the indicated phospholipids, and drugs were added to the aqueous subphase at the indicated concentration. Then titration with FP was carried out. Data were corrected to $\pi_{i}=24 \mathrm{mN} \cdot \mathrm{m}^{-1}$ and analyzed by non-linear, least squares regression to fit the equation $\Delta \pi=\Delta \pi_{\max } \cdot \mathrm{C} /\left(\mathrm{K}_{z}+\mathrm{C}\right)$.

\begin{tabular}{llll}
\hline Lipid & Drug & $\mathrm{K}_{z}(\mu \mathrm{M})$ & $\Delta \pi_{\max }\left(\mathrm{mN} \cdot \mathrm{m}^{-1}\right)$ \\
\hline DOPC & None & $0.55 \pm 0.04$ & $18.55 \pm 0.32$ \\
DOPC & Quinine $(1.6 \mu \mathrm{M})$ & $0.51 \pm 0.05$ & $20.45 \pm 0.50$ \\
DOPC & Mefloquine $(2.6 \mu \mathrm{M})$ & $0.53 \pm 0.06$ & $29.85 \pm 0.92$ \\
DOPC/PS & None & $0.97 \pm 0.07$ & $13.43 \pm 0.39$ \\
DOPC/PS & Quinine $(3.2 \mu \mathrm{M})$ & $0.19 \pm 0.14$ & $14.87 \pm 2.00$ \\
DOPC/PS & Mefloquine $(3.4 \mu \mathrm{M})$ & $0.43 \pm 0.09$ & $27.15 \pm 1.12$ \\
\hline
\end{tabular}

from the expected effect of an amphipatic cationic drug on the negatively charged membrane [10]. MQ also had a substantial effect on $\Delta \pi_{\max }$.

The above conclusions are further supported by a series of comparative experiments, where a fixed concentration of drug $(1 \mu \mathrm{M})$ and thereafter FP $(0.526 \mu \mathrm{M})$ were added to the monolayer aqueous subphase, and $\Delta \pi$ was measured. In the presence of $C Q$, sufficient time was allowed $(4-5 \mathrm{~h})$ to reach a constant level of surface pressure. Results shown in Table IV indicate that MQ is the most effective additive to induce FP dependent $\Delta \pi$ increase in all types of monolayers; next comes $Q$ and the least effective is CQ. The greatest relative enhancements of $\Delta \pi$ are

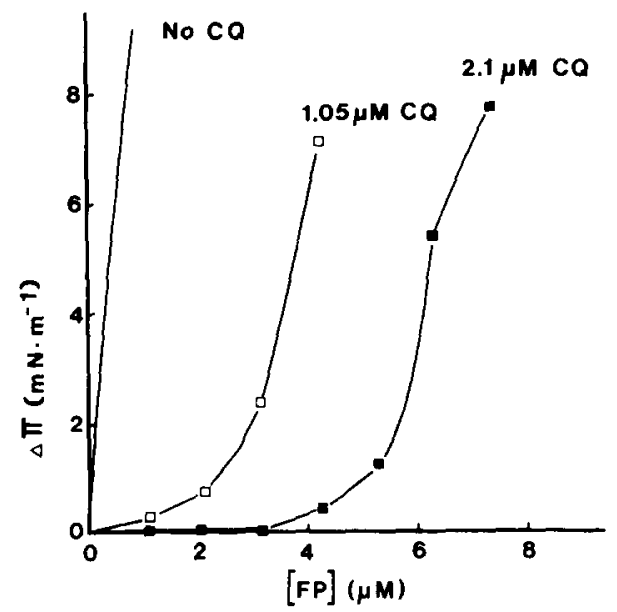

Fig. 2. Effect of FP on $\Delta \pi$ in CQ-doped DOPC monolayers. Monolayers were formed and CQ was introduced into the aqueous subphase. Then FP was added in increasing amounts and $\Delta \pi$ was measured. 
TABLE IV

RELATIVE EFFECTS OF DRUGS AND FP ON $\Delta \pi$ OF MONOLAYERS COMPOSED OF DIFFERENT PHOSPHOLIPIDS

N.D., not determined. Monolayers were formed, and drug at $1 \mu \mathrm{M}$ and then FP at $0.526 \mu \mathrm{M}$ were added to the aqueous subphase. All results were normalized to $\pi_{l}=24 \mathrm{mN} \cdot \mathrm{m}^{-1}$. The relative effect of the drug vs. non-doped membrane is shown as the ratio (R) of $\Delta \pi$ 's. Results shown are means of 3 experiments and the standard deviations were within $15 \%$ of the means.

\begin{tabular}{|c|c|c|c|c|c|c|c|c|c|c|c|c|}
\hline \multirow[t]{2}{*}{ Drug } & \multicolumn{2}{|c|}{ DOPC } & \multicolumn{2}{|c|}{$\begin{array}{l}\text { DOPC/PS } \\
(1: 1)\end{array}$} & \multicolumn{2}{|c|}{$\begin{array}{l}\mathrm{DOPC} / \mathrm{PI} \\
(1: 1)\end{array}$} & \multicolumn{2}{|c|}{$\begin{array}{l}\text { DOPC/ } \\
\text { CHOL } \\
(2: 1)^{\mathrm{a}}\end{array}$} & \multicolumn{2}{|c|}{ DLPC } & \multicolumn{2}{|c|}{$\mathrm{DPPC}^{\mathrm{b}}$} \\
\hline & $\Delta \pi$ & $\mathbf{R}$ & $\Delta \pi$ & $\mathbf{R}$ & $\Delta \pi$ & $\mathbf{R}$ & $\Delta \pi$ & $\mathbf{R}$ & $\Delta \pi$ & $\mathbf{R}$ & $\Delta \pi$ & $\mathbf{R}$ \\
\hline Control & 8.5 & 1.0 & 4.8 & 1.0 & 6.7 & 1,0 & 8.9 & 1.0 & 7.3 & 1.0 & 4.5 & 1.0 \\
\hline Chloroquine & 11.9 & 1.4 & 8.6 & 1.8 & 1.6 & 0.2 & 15.3 & 1.7 & 1.4 & 0.2 & 8.7 & 1.9 \\
\hline Quinine & 14.0 & 1.7 & 11.6 & 2.4 & 9.4 & 1.4 & 12.5 & 1.4 & \multicolumn{2}{|c|}{ N.D. } & \multicolumn{2}{|c|}{ N.D. } \\
\hline Mefloquine-1 & 18.5 & 2.2 & 14.9 & 3.1 & 13.7 & 2.0 & 16.4 & 1.9 & 15.5 & 2.1 & 10.7 & 2.4 \\
\hline Mefloquine-2 & 14.6 & 1.7 & 15.0 & 3.1 & 12.4 & 1.9 & 13.2 & 1.5 & & D. & & D. \\
\hline
\end{tabular}

${ }^{\mathrm{a}} \mathrm{FP}=0.263 \mu \mathrm{M}$.

${ }^{b_{F P}}=0.105 \mu \mathrm{M}$.

obtained with DOPC-PS monolayers, emphasizing the importance of surface charge in the interaction of the drug and/or FP with the monolayer. However, net surface charge in itself is not sufficient to account for the effect, as reflected in the reactivity of DOPC-PI monolayers, which probably means that the spatial distribution of charge may also be important. With highly unsaturated phospholipids (DOPC-PI and DLPC), MQ and Q increase the effect of FP but CQ decreases it. The maximal absolute effect of FP on $\Delta \pi$, both in control and doped monolayers, is obtained in density packed membranes, namely, those composed of DOPC-CHOL and of DLPC (note the FP concentrations used in these experiments). Comparison of values presented in Tables III and IV shows that in DLPC monolayers increase of drug concentration decreases the effect of FP, while with DOPC-PS membranes no such effect was observed.

Addition of preformed FP-drug complexes to the subphase (Table V) reveals effects which are sometimes different from those observed during the sequential addition of drug and FP. Some of these differences are certainly due to the different relative concentrations of drugs and hemin in these two different experimental designs. With DOPC, CQ decreases both $K_{z}$ (in terms of [FP]) and $\Delta \pi_{\max }$. FP.Q increases $K_{z}$ and $\Delta \pi_{\max }$, suggesting that the complex in the aqueous phase has a dissociation constant in the concentration range used, inasmuch as the free FP concentration is reduced, thus causing the apparent increase in $K_{z}$. Results obtained with FP-MQ are more ambiguous, since we do not have an explanation for the lack of effect on $\Delta \pi_{\max }$ in comparison with the dramatic effect observed when drug and FP are added sequentially (Table III). It is possible that true equilibrium 


\section{TABLE V}

EFFECT OF PREFORMED FP-DRUG COMPLEXES ON $\Delta \pi$ OF DIFFERENT TYPES OF MONOLAYERS

FP and drugs were mixed as $0.5 \mathrm{mM}$ stock solutions. Aliquots were added sequentially to the aqueous subphase and the resulting $\pi$ 's were recorded. Data of $\Delta \pi$ (normalized to $\pi_{i}=24$ $\mathrm{mN} \cdot \mathrm{m}^{-1}$ ) vs. complex concentration $\mathrm{C}$ (in terms of [FP]) were analyzed by means of nonlinear, least squares regression of the first order reaction $\Delta \pi=\Delta \pi_{\max } \cdot \mathrm{C} /\left(\mathrm{K}_{z}+\mathrm{C}\right)$.

\begin{tabular}{lllll}
\hline Lipid & Drug & FP-Drug & $\mathrm{K}_{z}(\mu \mathrm{M})$ & $\Delta \pi_{\max }\left(\mathrm{mN} \cdot \mathrm{m}^{-1}\right)$ \\
\hline DOPC & None & - & $0.55 \pm 0.04$ & $18.6 \pm 0.3$ \\
& Chloroquine & $1: 1$ & $0.47 \pm 0.03$ & $12.7 \pm 0.5$ \\
& Quinine & $1: 1$ & $1.01 \pm 0.07$ & $22.3 \pm 0.6$ \\
& Mefloquine & $1: 1$ & $0.53 \pm 0.02$ & $18.5 \pm 0.2$ \\
DOPC-PS & None & - & $0.97 \pm 0.07$ & $13.4 \pm 0.4$ \\
& Chloroquine & $1: 1$ & $0.17 \pm 0.01$ & $11.8 \pm 0.2$ \\
& Chloroquine & $2.5: 1$ & $0.16 \pm 0.05$ & $12.6 \pm 0.6$ \\
& Quinine & $1: 1$ & $0.45 \pm 0.03$ & $16.6 \pm 0.3$ \\
& Mefloquine & $1: 1$ & $0.34 \pm 0.02$ & $12.0 \pm 0.2$ \\
& & & & \\
DOPC-PI & None & - & $0.39 \pm 0.06$ & $12.3 \pm 0.4$ \\
& Chloroquine & $2.5: 1^{\mathrm{a}}$ & $0.09 \pm 0.01$ & $10.2 \pm 0.1$ \\
\hline
\end{tabular}

${ }^{\mathrm{a}}$ The best fit was obtained when $C$ was raised to the power of $1.77 \pm 0.02$.

has not been achieved in these experiments or that some irreversible processes have taken place.

In DOPC-PS monolayers titration with performed complexes results in a substantial decrease in $K_{z}$ but only small effects on $\Delta \pi_{\max }$. Adsorption of the complex to the negatively charged monolayer could account for these observations.

\section{Discussion}

Hemin (FP) is known to interact with biological membranes $[1,3]$ as do other porphyrins [30]. Such interactions are primarily mediated by the lipid component of membranes in as much as FP binds to phospholipid bilayers [8,31]. Binding of FP involves interactions with both the headgroup and the hydrocarbon chain of the lipid; hence, it is very likely that the iron atom and the negatively charged propionic side chains of hemin reside in the polar head group region, while its dimethyl-divinyl side is immersed in the hydrocarbon region. Phospholipids are able to bind FP at 4:1 molar ratios [8] and red cell membranes do so up to $1 \mathrm{FP}$ molecule per phospholipid [3]. In the saturating concentration range, FP is able to cross both phospholipid and cell membranes.

In the present report we have shown that FP interacts with phospholipid mono- 
layers composed of different lipids, inducing an increase in surface pressure. We suggest that this effect is due to the intercalation of FP into the monolayer.

The relationship between the different parameters characterizing the intercalation of FP into monolayers made of various phospholipids and $\pi_{i}$ was evaluated as follows: data presented in Table I were used to generate a $\Delta \pi$ vs. [FP] relationship for each $\pi_{i}$ chosen (Eqn. 2). The generated data were analyzed according to Eqn. (3). Figure 3 displays the dependence of the power $(z)$ to which [FP] has to be raised in order to get the best fit of the data to Eqn. (3). The power is an indicator of the average stoichiometry of the FP intercalating species. From this figure it is clear that as $\pi_{i}$ increases, the power decreases.

Since FP exists mostly as dimers at the FP concentration range used [32], it can be concluded from the power dependence that when the monolayer is expanded (low $\pi_{i}$ ), FP is able to intercalate as a dimer, and when the monolayer is compressed, the power monotonically decreases, until finally, at $\pi_{i}$-values com. parable to those estimated for erythrocyte membranes [24], it approaches 1, implying that FP intercalates as a monomer. The largest differences between the various phospholipids are observed in expanded monolayers and, as $\pi_{i}$ is raised, these differences vanish. An opposite picture is observed when DOPC is compared with DOPC-CHOL. This behavior is consistent with the packing effect of cholesterol [25]. At low $\pi_{i}$, FP has the easiest access as a dimer into DOPC-PI mono. layers, probably due to the source of PI (yeast) used in this investigation, which has a high content of unsaturated acyl chains [33]. At the other end, DOPC-PS monolayers accomodate mostly monomers, probably due to charge repulsion.

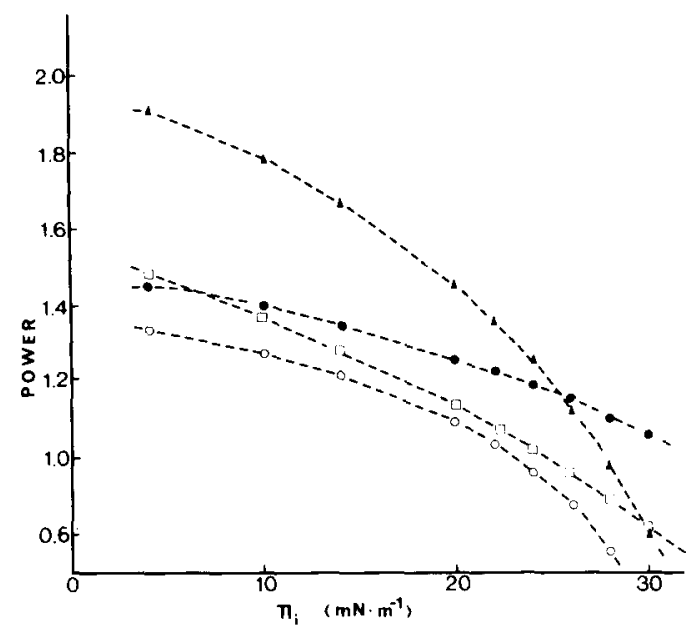

Fig. 3. Dependence of the stoichometry of the FP intercalating species $(z)$ on $\pi_{i}$. Experimental data from Table I were used to generate $\Delta \pi$ vs. [FP] at different $\pi_{i}$ (Eqn. 2). Values of $z$ were retrieved by non-linear, least squares analysis to fit Eqn. 3. •, DOPC; 4 , DOPC/PI $(1: 1) ; 0$, DOP $(1: 1) ; \square$, DOPC/CHOL $(2: 1)$. 
Comparison of PI and PS containing monolayers strongly suggests that molecular packing is more important than electrostatic forces in governing the intercalation of FP.

The same conclusions can be reached when the half-saturation constants of Eqn. (3), $\mathrm{K}_{z}$, are related to $\pi_{i}$ (Fig. 4). For all types of monolayers $\mathrm{K}_{z}$ rises exponentially with $\pi_{i}$. Such behavior implies that higher free energies are required for intercalation of FP into compacted monolayers. This could result both from denser packing and an increase in the surface potential in monolayers composed of acidic phospholipids. Accordingly, FP has an easier access into DOPC-PI (as compared with DOPC) monolayers because of difference in packing, and $K_{z}$ is invariably the largest with DOPC-PS monolayers at all levels of $\pi_{i}$. Cholesterol increases the apparent affinity of the monolayer to FP, as a result of the maximal packing in these monolayers, since binding of FP to phospholipid bilayers is reduced in the presence of cholesterol [8]. Interestingly, if we extrapolate $\mathrm{K}_{z}$ to the estimated

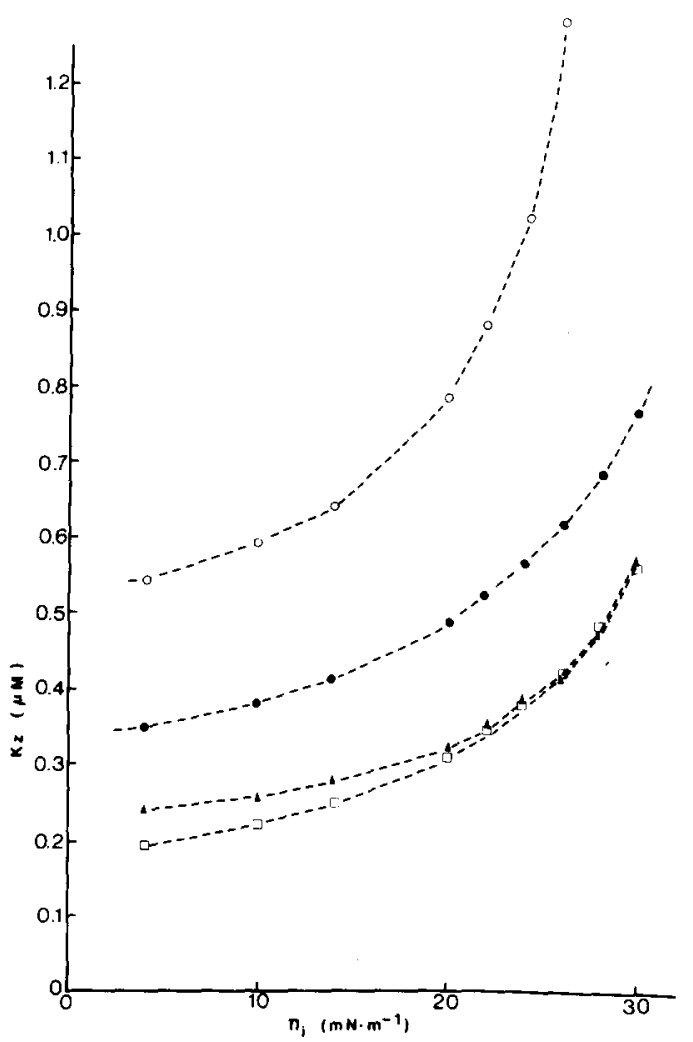

Fig. 4. Dependence of the half saturation constant $K_{z}$ of FP effect on $\Delta \pi$, on $\pi_{i}$. Experimental data from Table I were used to generate $\Delta \pi$ vs. [FP] at different $\pi_{i}$ (Eqn. 2). The values of $\mathrm{K}_{z}$ were retrieved by non-linear, least squares analysis according to Eqn. (3). Symbols are identical to those of Fig. 3. 
surface pressure of erythrocytes [24], we arrive at FP concentrations which are known to cause lysis of erythrocytes [1, 3; Ginsburg \& Azolay, unpublished]. This result further emphasizes the notion that phospholipids are the primary target for FP effect on cell membranes.

As it is generally observed [34], $\Delta \pi_{\max }$ decreases with increasing $\pi_{i}$ (Fig. 5), and the total surface pressure at each $\pi_{i}$, e.g., the sum of $\Delta \pi_{\max }$ and $\pi_{i}$, almost reaches the collapse pressure of the monolayer. The largest differences of $\Delta \pi_{\max }$ are found at high $\pi_{i}$ between DOPC-PI and DOPC-CHOL monolayers. It is at these high $\pi_{i}$ that the greatest packing order is achieved in the presence of cholesterol and each intercalating FP molecule produces a maximal effect on the surface pressure, while even in condensed DOPC-PI monolayers there is sufficient free space to allow FP intercalation with a minor effect on $\Delta \pi$. This result implies that cell membranes composed of relatively unsaturated phospholipids should be less susceptible to the lytic effect of FP [9] and probably underlies the differential effect of FP on cell membranes which differ in their lipid composition. Moreover, Tipping et al. [8] suggested that FP may form aggregates in phospholipid membranes. Such aggregation may be affected by the nature of the phospholipids

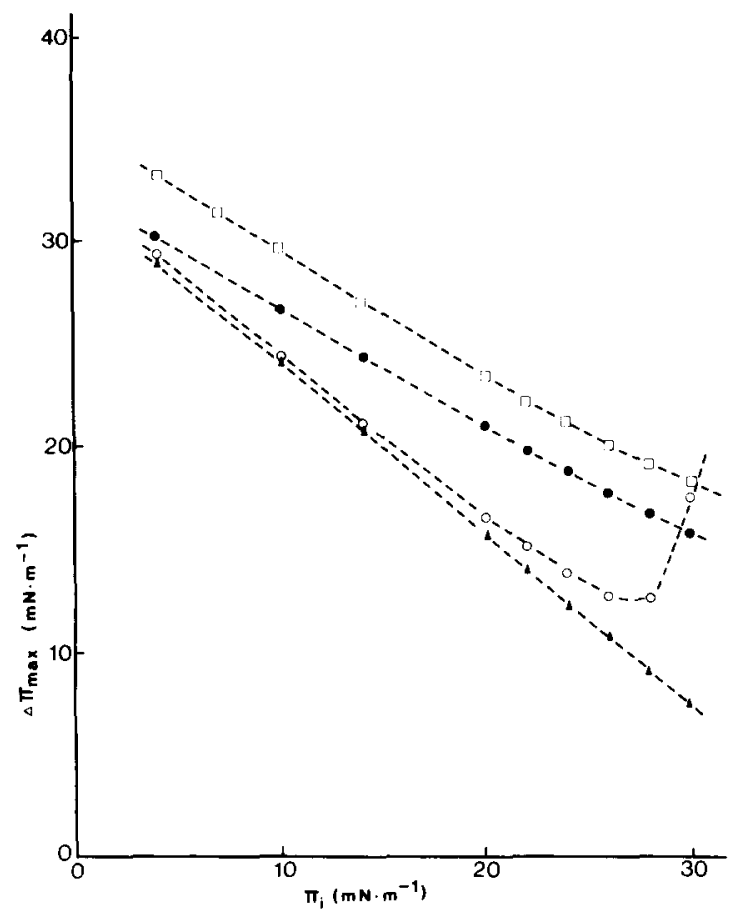

Fig. 5. Dependence of $\Delta \pi_{\max }$ on $\pi_{i}$. Experimental data from Table I were used to generate $\Delta \pi$ vs. [FP] at different $\pi_{i}$ (Eqn. 2). Values of $\Delta \pi_{\max }$ were retrieved by non-linear, least squares analysis to fit Eqn. (3). Symbols are identical to those in Fig. 3. 
composing the membrane and, in turn, affect the solubilization of FP into the membrane and the surface pressure.

Considering erythrocyte membranes where the phospholipids are distributed asymmetrically [36] and the apparent ability to FP to cross cell membranes [3] and phospholipid bilayers [8], FP is expected to act primarily on the outer monolayer because it has more cholesterol and no phosphatidylserine. Such interaction could perturb the membrane structure and cause lysis. However, it is questionable whether this is relevant to physiological conditions, since FP binds to both plasma proteins [37] and erythrocyte cytosolic component(s) [26] with a similar or greater affinity than its binding to phospholipids. Therefore, under physiological conditions, unless FP is present in very large amounts as it is delivered by direct interaction of its protein carrier with the membrane [31], the chances that it would cause membrane damage are very remote. This is probably the reason why FP, even at high concentrations, does not damage erythroid cells grown in serumcontaining media (see, for example, Ref. 6).

The interaction of antimalarial drugs with phospholipids has received some attention recently because some of these drugs are also lysosmotropic and cause lipidosis $[10,15]$. This latter effect could be the result of phospholipase inactivation [38], or, alternatively, the direct interaction of the drug with phospholipids [12] could render the substrate unavailable to the enzyme. Of the three drugs tested in the present work, both $\mathrm{Q}$ and MQ had an effect on surface pressure, and more so when the monolayer was negatively charged. CQ demonstrably bind to acidic and neutral phospholipids $[8,11,13]$ but has no effect on $\Delta \pi$. This observation must result from the nature of the drug's side chain which is considerably more polar and charged than those of MQ and Q, and is consistent with the poor miscibility of CQ in organic phases, even when complexed with FP (Ginsburg and Blauer, unpublished observations). Direct binding measurements in the monolayer system could ascertain these conclusions.

The combined effect of drugs and FP on $\Delta \pi$ is very complex and results from the multi-equilibrium situation exemplified in Fig. 6. Different processes depicted by this scheme may be rate-limiting, hence explaining the effect of the order of addition of FP and drug on $\Delta \pi$. It is possible that this latter effect implies that a true thermodynamic equilibrium was not achieved in all experimental systems. Longer equilibration times are nevertheless impractical because the observed

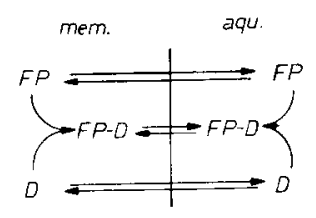

Fig. 6. Possible modes of distribution of FP, drug (D) and FP-drug complexes in phospholipid membranes. 
phenomena could be compromised by various non-specific processes. However, since the lytic effect of FP and drugs on biological membranes has a time constant of a few minutes, i.e. very similar to the major effect that was measured, even quasi steady state results are relevant for the elucidation of the underlying mechanism. The situation is further complicated by the following considerations: the total amounts of phospholipids $(\sim 9 \mathrm{nmol})$ and ligands $(1-40 \mathrm{nmol})$ present in the system are of the same order of magnitude, but since all the lipids are organized in the monolayer, their concentration is much larger $\left(10^{6}\right.$-fold $)$ than that of the ligand. Hence, if the derived $\mathrm{K}_{z}$-values are similar to the actual dissociation constants, it follows that most of the ligand is bound to the monolayer and it is not surprising that addition of drug after FP had barely any effect on $\Delta \pi$, except for $M Q$. This result is in marked contrast to the potentiating effect of $C Q$ and $Q$ on FP induced lysis of erythrocytes [1], suggesting that the asymmetric distribution of lipids and the presence of an intracellular FP receptors may affect the distribution of FP and drugs as well as their subsequent interaction and effect on membrane integrity. This discrepancy emphasizes the limitation of model systems in simulating the behavior of biological membranes.

When drug is added first and then FP, a marked increase in $\Delta \pi$ is observed (Table IV). This is mainly due to reduction of $\mathrm{K}_{z}$ in DOPC-PS monolayers, as would be expected from drug effect on surface charge and an increase in $\Delta \pi$ in DOPC monolayers (Table III), suggesting a different mode of intercalation and/or aggregation of FP in drug-modified monolayers. The exceptionally large effect of MQ on $\Delta \pi_{\max }$ is consistent with its demonstrably strong interaction with FP in apolar environment [18].

The effect of CQ in this system differs markedly from that of the other drugs, in as much as $t_{1 / 2}$ for equilibration is much longer and, in monolayers of unsaturated phospholipids, it reduces the effect of FP. These results are consistent with the fact that the apparent affinity of FP for CQ is 10-100 times larger than for MQ and $Q$ [39], thus providing for the sequestration of most FP in multimer complexes with CQ [29] in the aqueous phase with only slow release of FP to monolayers, provided they are sufficiently hydrophobic [9]. The large FP-CQ aqueous complex like amphipatic proteins [34], is probably unable to penetrate into the monolayer as such.

The study of the effect of preformed FP-drug complexes was undertaken because of its implication for antimalarial activity. Fitch [16] suggested that CQ forms a membrane toxic complex with FP, which is produced during hemoglobin digestion by the malarial parasite, and that unlike FP alone, this complex is not sequestered by plasma or cytoplasmic ligands. The present results (Table V) are consistent with this hypothesis, as all preformed complexes have, indeed, a substantial effect on $\Delta \pi$. Since with acidic phospholipids the effect of the various complexes is substantially increased due to a decrease in $K_{z}$, we suggest that the complexes adsorb to the monolayer interface and release FP to the phospholipid through the formation of a continuous hydrophobic phase. This mechanism could 
explain the largest reduction of $\mathrm{K}_{\mathbf{z}}$ observed with the most stable FP-CQ complex in monolayers containing PS and PI. On the other hand, the smaller affinity of $Q$ and MQ for FP may preclude the formation of complexes in the presence of cellular FP ligands, implying that the mode of antimalarial action of these drugs could be altogether different from that of CQ. Furthermore, MQ is 100 times more active than $Q$ as an antimalarial drug $[20,40]$. Such difference in activity is inconsistent with the similar effects that these two drugs exert on phospholipid monolayers. Further investigations are needed in order to clarify the precise mode of action of quinine and mefloquine.

\section{Acknowledgements}

This work was supported by the UNDP/World Bank/WHO Special Programme for Research and Training in Tropical Diseases and by an EMBO Short-Term Fellowship to H.G.

\section{References}

1 A.C. Chou and C.D. Fitch, J. Clin. Invest., 66 (1980) 856- 858 .

2 A.C. Chou and C.D. Fitch, J. Clin. Invest., 68 (1981) 672-677.

3 I. Kirschner-Zilber, E. Rabizadeh and N. Shaklai, Biochim. Biophys. Acta, 690 (1982) $20-30$.

4 A.U. Orjih, H.S. Banyal, R. Chevli and C.D. Fitch, Science, 214 (1981) 667-669.

5 C.D. Fitch, R. Chevli, H.S. Banyal, G. Phillips, M.A. Pfaller and D.J. Krogstad, Antimicrob. Agents Chemother., 21 (1982) 819-822.

6 T.J. Dougherty, J.E. Kaufman, A. Goldfarb, K.R. Weishaupt, D. Mittleman and A. Mittleman, Cancer Res., 38 (1978) 2628-2635.

7 E. Tipping, B. Ketterer, L. Christodoulides and G. Enderby, Biochem. J., 157 (1976) $461-467$.

8 E. Tipping, B. Ketterer and L. Christodoulides, Biochem. J., 180 (1979) 327-337.

9 H. Ginsburg and R.A. Demel, Biochim. Biophys. Acta, 732 (1983) 316-319.

$10 \mathrm{H}$. Lüllmann, R. Lüllmann-Rauch and O. Wassermann, Biochem. Pharmacol., 27 (1978) $1103-1108$.

11 A. Harder, S. Kovatchev and H. de Buch, Hoppe-Seyler's Z. Physiol. Chem., 361 (1980) $1847-1850$.

12 C.A. Dise, J.W. Busch and D.B.P. Goodman, J. Biol. Chem., 257 (1982) 4701-4704.

13 R. Chevli and C.D. Fitch, Antimicrob. Agents Chemother., 21 (1982) 581-586.

14 H. Lüllmann, H. Plösch and A. Ziegler, Biochem. Pharmacol., 29 (1980) 2969-2974.

15 A. Yamamoto, S. Adachi, Y. Matsuzawa, I. Kitani, A. Hiraoka and K. Seki, Lipids, 11 (1975) 616-622.

16 C.D. Fitch, Malaria and the Red Cell, Ciba Foundation Symposium 94, Pitman, London, 1983, pp. 222-232.

17 S.N. Cohen, K.O. Phifer and K.L. Yielding, Nature, 202 (1964) 805-806.

18 D.C. Warhurst, Biochem. Pharmacol., 30 (1981) 3323-3327.

19 W. Peters, Br Med Bull., 38 (1982) 187-192.

20 T.G. Geary and J.B. Jensen, J. Parasitol., 69 (1983) 97-105.

21 R.A. Demel, Methods Enzymol., 32B (1974) 539--545.

22 L.L.M. van Deenen and G.H. de Haas, Adv. Lipid Res., 2 (1964) 167-234. 
23 T.G. Warner and A.A. Benson, J. Lipid Res., 18 (1977) 548-552.

24 L.L.M. van Deenen, R.A. Demel, W.S.M. Geurts van Kessel, H.H. Kamp, B. Roelofsen, A.J. Verkleij, K.W.A. Wirtz and R.F.A. Zwaal, in: Y. Hatefi and I. Djavadi-Ohaniance (Eds.), Structural Basis of Membrane Function, 1976, pp. 21-38.

25 R.A. Demel and B. de Kruyff, Biochim. Biophys. Acta, 457 (1976) 109-132.

26 H.S. Banyal and C.D. Fitch, Life Sci., 13 (1982) 1141-1144.

27 A. Jearnpipatkul and B. Panijpan, Chem.-Biol. Interact., 33 (1980) 83-90.

28 S. Moreau, B. Perly and J. Biguet, Biochimie, 64 (1982) 1015-1025.

29 G. Blauer and H. Ginsburg, Biochem. Int., 5 (1982) 519-523.

30 T.J. Dougherty and R.E. Thoma, in: R. Pratesi and C.A. Sacchi (Eds.), Photomedicine and Photobiology, Springer Verlag, Berlin, 1980, p. 67-85.

31 Y. Shviro, I. Zilber and N. Shaklai, Biochim. Biophys. Acta, 687 (1982) 63-70.

32 S.B. Brown, T.C. Dean and P. Jones, Biochem. J., 117 (1970) 733-739.

33 W.E. Trevelyan, J. Lipid Res., 7 (1966) 445-447.

34 P.J. Quinn and R.M.C. Dawson, Biochem. J., 113 (1969) 791-803.

35 W.S.M. Geurts van Kessel, M. Tieman and R.A. Demel, Lipids, 16 (1981) 58-63.

36 J.A.F. Op den Kamp, Annu. Rev. Biochem., 48 (1979) 47-71.

37 O. Walker, D.J. Birkett, G. Alvan, L.L. Gustafsson and F. Sjögvist, Br. J. Clin. Pharmacol., 15 (1982) 375-377.

38 Y. Matsuzawa and K.Y. Hostetler, J. Biol. Chem., 225 (1980) 5190-5194.

39 A.C. Chou, R. Chevli and C.D. Fitch, Biochemistry, 19 (1980) 1543-1546.

40 T.R. Sweeney, Med. Res. Rev. (1981) $284-301$. 